Objectives The aim of the present study was to determine whether adult CF patients with pulmonary NTM infection have worse clinical outcomes, when compared to a matched group of $\mathrm{CF}$ patients who have never had NTM isolated. Additionally, the study also aims to determine what proportion of the overall bacterial community is NTM compared to other bacteria.

Method Clinical data were collected from 12 spontaneously expectorating CF patients who cultured $M$ abscessus in the sputum since they were first infected with $M$ abscessus, and 24 matched controls who have CF, but never been infected with $M$ abscessus. Student unpaired t-test was used to compare the mean forced expiratory volume $\left(\mathrm{FEV}_{1}\right)$ per year between the two groups.

Results Data from 36 individuals were studied. Cases and controls were well matched. Mean absolute decline in $\mathrm{FEV}_{1}$ was $100 \mathrm{ml} /$ year $(\mathrm{SD} \pm 0.19)$ in cases and $79 \mathrm{ml} /$ year $(\mathrm{SD} \pm 0.19)$ in controls. Student unpaired t-test was not significant (0.73). Mean absolute percentage predicted decline in $\mathrm{FEV}_{1}$ was $3.85 \%$ (SD \pm 5.62 ) in cases and $2.78 \%$ $(\mathrm{SD} \pm 6.58)$ in controls. Student unpaired t-test was not significant (0.62).

Conclusion These results indicate that there is a small decline in mean $\mathrm{FEV}_{1}$ per year between the two groups; $M$ abscessus and the controls, but the difference is statistically and clinically insignificant.

\section{P166 TEMPORAL DYNAMICS OF POLYMICROBIAL COMMUNITIES IN THE LOWER RESPIRATORY TRACT OF PATIENTS WITH CYSTIC FIBROSIS}

doi:10.1136/thoraxjnl-2011-201054c.166

${ }^{1} \mathrm{~A}$ Nelson, ${ }^{2} \mathrm{~S} J$ Bourke, ${ }^{3} \mathrm{~J} \mathrm{D}$ Perry, ${ }^{4} \mathrm{~A}$ De-Soyza, ${ }^{1} \mathrm{~S}$ P Cummings. ${ }^{1}$ Northumbria University, Newcastle upon Tyne, UK; ${ }^{2}$ Royal Victoria Infirmary, Newcastle upon Tyne, UK; ${ }^{3}$ Freeman Hospital, Newcastle upon Tyne, UK; ${ }^{4}$ Newcastle University, Newcastle upon Tyne, UK

Introduction and Objectives The microbial communities present in CF sputum are polymicrobial and consist of bacteria, viruses and fungi. Although stratified studies have demonstrated a change in the CF bacterial microbiota with increasing age, individual patients have not been followed longitudinally across stable phases and exacerbation episodes period of time. The aim of this study was to follow CF patients who were either homo- or heterozygous for the F508del mutation over a period of up to 20 months to assess how the bacterial and fungal communities fluctuate over this period to determine whether a shift in the microbiota could be linked with acute pulmonary exacerbations.

Methods Adult CF patients were recruited and spontaneously expectorated sputum samples were collected. DNA was extracted from the samples and PCR-DGGE was used to analyse the bacterial and fungal communities using universal primer sets.

Results Routine microbial culture identified a mean of 1.52 bacterial species and 0.62 fungal species, whereas molecular analysis found a mean of 12.24 bacterial species and 1.41 fungal species across the cohort. The composition of the bacterial communities between patients varied significantly according to gender and being culture positive for $P$ aeruginosa. Patients homozygous for the F508del mutation had more rich fungal communities than heterozygotes. However, a bacterial or fungal community characteristic for pulmonary exacerbations was not observed.

Conclusions Our data clearly demonstrates that bacterial and fungal communities in the CF lower respiratory tract are more diverse than previously thought. Furthermore, the microbial communities in the lower respiratory tract of CF patients are subject are selected by predisposing factors such as gender but still remain unique to individual patients. Monitoring the microbial communities has found that although they are subject to some fluctuation a characteristic community does not assemble to cause acute pulmonary exacerbations. Furthermore, particular bacterial taxa were present throughout the sampling period, suggesting that current antimicrobial therapies are not adequate at removing these taxa.

\section{P167 SCREENING FOR VIRAL UPPER RESPIRATORY TRACT INFECTION IN PULMONARY EXACERBATIONS IN CYSTIC FIBROSIS}

doi:10.1136/thoraxjnl-2011-201054c.167

R J José, S Hassan, M Butler, D Watson, L Kuitert. Adult Cystic Fibrosis Service, London Chest Hospital, Barts and the London NHS Trust, London, UK

Introduction and objectives It is unclear if respiratory viruses are important in precipitating pulmonary exacerbations in adults. Respiratory viruses are considered important pathogens in children with cystic fibrosis (CF), as they may be associated with deterioration in lung function and bacterial infection. ${ }^{12}$ Following the "flu" pandemic in 2009 we have started screening CF patients for viral upper respiratory tract infection for infection control. Our aim was to determine if adult CF patients admitted with a pulmonary exacerbation had positive nasopharyngeal swabs suggesting a viral aetiology.

Methods Retrospective review of admissions to an adult CF unit between May 2009 and May 2011 to identify those who had nasopharyngeal swabs (NPS) for the molecular detection (PCR) of viruses in the nasopharyngeal tract. Data were collected from the hospitals' computer information system. Continuous variables are described as median averages (IOR), and categorical variables as counts.

Results 365 admissions were identified during this period (93 patients). 299 admissions were due to pulmonary exacerbation. A NPS was performed on 211 admissions (174 due to pulmonary exacerbation). Characteristics of patients that had NPS on admission are listed in Abstract P167 table 1. Only 5 (2.9\%) NPS detected upper respiratory tract viruses in patients with a pulmonary exacerbation. Influenza A [H1N1] and Parainfluenzae were detected in three and two NPS respectively.

Abstract P167 Table 1 Characteristics of patients at admission-for patients with nasopharyngeal swabs

\begin{tabular}{lll}
\hline & $\begin{array}{l}\text { Pulmonary } \\
\text { exacerbation }(\mathbf{n}=174)\end{array}$ & $\begin{array}{l}\text { Non-pulmonary } \\
\text { exacerbation }(\mathbf{n}=\mathbf{3 7})\end{array}$ \\
\hline Age (years) & $22(6)$ & $21(5)$ \\
FEV $1 \%$ predicted) & $58(34)$ & $70(31)$ \\
FVC (\% predicted) & $76(32)$ & $91(32)$ \\
Length of stay (days) & $13(8)$ & $5(11)$ \\
White cell count $\left(\times 10^{9} /\right)$ & $9(5)$ & $8(2)$ \\
C-reactive protein $(\mathrm{mg} / \mathrm{l})$ & $14(28)$ & $5(11)$ \\
Virus NPS positive & 5 & 0 \\
\hline
\end{tabular}

Conclusions Viral infection, detected by NPS, is not common in adult CF patients admitted to our hospital with or without pulmonary exacerbations. Screening all adult patients with CF admitted to hospital for the presence of viruses in the upper respiratory tract has a low yield and is not recommended.

\section{REFERENCES}

1. Wat $\mathbf{D}$, Gelder $\mathrm{C}$, Hibbitts $\mathrm{S}$, et al. The role of respiratory viruses in cystic fibrosis. $J$ Cyst Fibros 2008;7:320-8.

2. Conway SP, Simmonds EJ, Littlewood JM. Acute severe deterioration in cystic fibrosis associated with infuenza A virus infection. Thorax 1992;47:112-14.

\section{P168 ERADICATION OF BURKHOLDERIA CEPACIA IN CF: TIME FOR A COORDINATED APPROACH?}

doi:10.1136/thoraxjnl-2011-201054c.168

${ }^{1} \mathrm{~A}$ Horsley, ${ }^{2} \mathrm{~J}$ Govan, ${ }^{1} \mathrm{~A}$ M Jones. ${ }^{1}$ Manchester Adult CF Centre, Manchester, UK; ${ }^{2}$ University of Edinburgh, Edinburgh, UK

Introduction New infection with Burkholderia cepacia complex (Bcc) organisms is a significant event for patients with cystic fibrosis (CF). In addition to potential clinical impact, there are implications for 
access to services and transplantation. Prior to developing a protocol for attempted eradication of Bcc, similar to that applied to Pseudomonas aeruginosa, UK CF centres were surveyed to establish what practices are currently employed.

Methods A questionnaire was distributed to CF centre directors and non-returns followed up by email reminder.

Results Responses were obtained from 35 units (73\%). Because of the rarity of new Bcc infection in paediatric centres, only replies from 16 adult centres (representing 3400 patients) have been analysed further. 12 centres, representing 2860 patients, always attempt eradication of newly isolated Bcc. Two additional centres attempt eradication only in the presence of additional indications. Only two units had a formal eradication policy. IV antibiotics were used in all cases, for a median of 2 (range 2-6) weeks, typically comprising combined tobramycin and meropenem with additional therapy consisting of septrin $(n=5)$, ceftazidime (6), and chloramphenicol (2). Nebulised antibiotics (typically tobramycin or meropenem) were also used in 13 of these 14 centres. Five units used additional oral antibiotics, for a median of 7 $(2-12)$ weeks. This most commonly involved minocycline $(n=4)$ and/or septrin $(\mathrm{n}=5)$. Two thirds of adult centres estimated success rate of eradication therapy to be $<50 \%$. In centres where eradication was not routine, factors that dissuaded clinicians were perceived poor success of treatment $(n=5)$, toxicity $(n=3)$, cost $(n=1)$ and lack of experience with this approach $(n=2)$.

Conclusions Attempted eradication of newly acquired Bcc is controversial, involving expensive and potentially toxic therapies with no evidence to guide treatment choice and no published outcome data. Despite this, it is common practice in many UK adult CF centres. Most units do not have a formal eradication policy, adopting a variety of approaches, and estimates of treatment success are pessimistic. Since new acquisition of Bcc is now relatively rare, it is hard for even large units to assess response to therapies. A systematic approach is required to optimise and standardise treatment regimens, and assess outcomes.

\section{P169 CHARACTERISTICS AND SURVIVAL OF PATIENTS WITHOUT CYSTIC FIBROSIS (CF), ISOLATING MUCOID P AERUGINOSA (MPA) IN SPUTUM SAMPLES}

\section{doi:10.1136/thoraxjnl-2011-201054c.169}

R Ahmed, M Denton, I Clifton, D Peckham. Leeds Teaching Hospitals NHS Trust, Leeds, UK

Introduction and Objectives It is well established in patients with CF $\mathrm{m} P A$ indicates chronic infection and is associated with increased morbidity and mortality. Mucoid PA can be isolated in people with non-CF lung disease but to-date no study has systematically characterised these patients. The aim of this study was to characterise and evaluate the significance of mucoid strain in a large non CF population

Methods This study retrospectively examined the demography, clinical diagnoses and survival, of patients without CF with an initial isolate of $\mathrm{mPA}$ from their respiratory samples, between January 2008 and December 2010, in Leeds. Data were obtained from the microbiology and radiology databases, and from the clinical notes. Ethical approval was obtained.

Results A total of 170 patients isolated $\mathrm{mPA}$ in sputum for the first time during the study period. The median (range) age was 73 (22-99) years and $54 \%$ were female. $46 \%$ of patients had samples obtained during an in-patient stay. $21 \%$ of the patients had never seen a respiratory physician. The majority of patients had a diagnosis of bronchiectasis (See Abstract P169 figure 1). A third of patents had isolated non-mucoid $P A$ prior to isolating $\mathrm{mPA}$. $62 \%$ of the patients had a subsequent respiratory sample analysed and the majority continued to grow $\mathrm{mPA}$. 24 patients also grew Methicillinresistant Staphylococcus aureus (MRSA) from their sputum. The median age (range) of death was 78 (40-95) years. The cumulative survival was $50 \%$ at 3 years after isolating $\mathrm{mPA}$. Factors associated with increased mortality included co-isolation of MRSA, no previous respiratory review, isolating $\mathrm{m} P A$ during in-patient care and absence of bronchiectasis $(p<0.05)$.

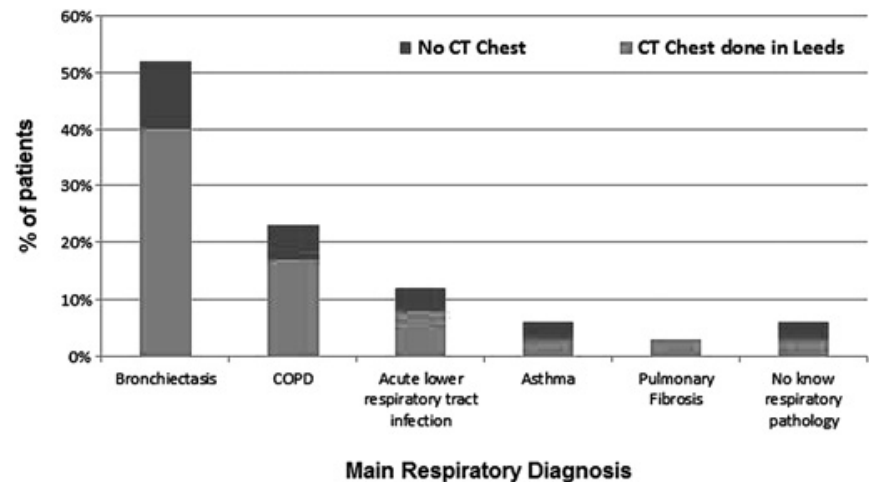

Abstract P169 Figure 1 Diagnosis of patients isolating $\mathrm{mPA}$.

Conclusions Mucoid $P$ aeruginosa isolated from the sputum of patients without CF may persist and is associated with high mortality. The underlying respiratory diagnosis of patients isolating $\mathrm{m} P A$ includes a range of respiratory diseases. There may be an opportunity to eradicated $P$ aeruginosa in patients who grow nonmucoid $P A$ before the emergence of $\mathrm{m} P A$.

\section{P170 A COST-EFFECTIVENESS ANALYSIS OF RESPIRATORY SYNCYTIAL VIRUS (RSV) PROPHYLAXIS IN INFANTS IN THE UK}

doi:10.1136/thoraxinl-2011-201054c.170

${ }^{1}$ A Bentley, ${ }^{2}$ Filipovic, ${ }^{2} \mathrm{~K}$ Gooch, ${ }^{2} \mathrm{~K}$ Buesch. ${ }^{1}$ Abacus International, Bicester, UK; ${ }^{2}$ Abbott Laboratories, Solna, Sweden

Background and Aim RSV is the most common cause of lower respiratory tract infection in young children and may result in hospitalisations, long-term sequelae, such as recurrent wheeze and/or asthma, and possibly death. Palivizumab is a prophylaxis against severe RSV infection and has been shown to reduce RSV-related hospitalisations, and provides a preventative option for high-risk infants. The aim of this study is to demonstrate the cost-effectiveness of RSV prophylaxis compared to no prophylaxis in the following high-risk infants groups: infants with chronic lung disease (CLD) and premature babies $(<29$, 29-32 and 33-35 weeks gestational age (wGA)).

Methods A decision-tree model was developed to reflect the clinical pathway of infants at high-risk of severe RSV infection. Baseline risk of RSV-hospitalisations and efficacy data were taken from palivizumab clinical trials and published meta-analysis. Cost data were obtained from national databases and published literature using an NHS perspective. The main outcome was presented as the incremental costs per Quality-Adjusted Life-Year (OALY) gained (ICER). The base-case analysis considered a lifetime horizon to capture the impact of long-term morbidity and mortality associated with RSV hospitalisations.

Results Prophylaxis against severe RSV infection resulted in ICERs of $£ 19168$, $£ 18174$ \& $£ 1185$ per OALY for high-risk infants with, CLD and the premature infant groups, $<29$ wGA and 29-32 wGA respectively compared to no prophylaxis. All results are below the accepted NICE threshold of $£ 30000$ /OALY thus demonstrating cost-effectiveness. The baseline ICER for the 33-35 wGA subgroup was above this threshold however, sensitivity analysis considering risk-factors in this subgroup showed that an increase in baseline risk of hospitalisation, from $7.2 \%$ to $11.24 \%$, led to palivizumab becoming a cost-effective option. 